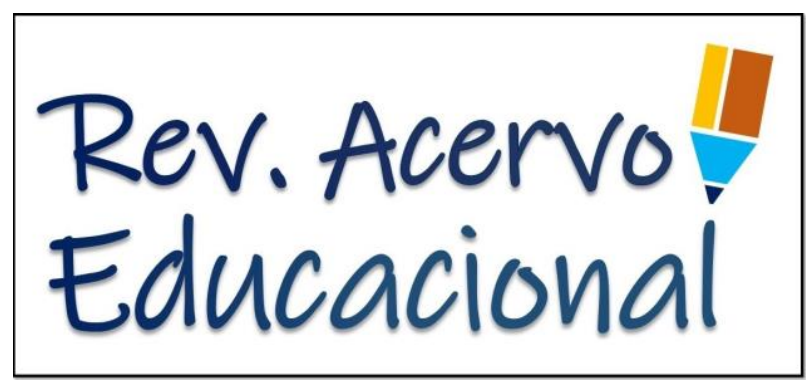

RELATO DE EXPERIÊNCIA

Recebido em: 5/2020

Aceito em: 6/2020

Publicado em: 9/2020

\title{
Relato de experiência: teoria especial da relatividade interação física- literatura em uma disciplina eletiva do ensino integral da Paraíba
}

\author{
Experience report: special theory of relativity physical-literature interaction in na elective \\ discipline of the integral education of Paraíba
}
Informe de experiencia: teoría especial de la relatividade interacción físico-literatura en una disciplina electiva de la educación integral de Paraíba

Rafael Leal da Silva ${ }^{1 *}$.

\begin{abstract}
Resumo: O presente trabalho tem a intenção de demonstrar o potencial didático na articulação entre física e literatura através de uma metodologia interdisciplinar como intercessão pedagógica estimulante nas aulas de física para alunos do ensino médio de uma escola pública estadual. Para tanto, foi desenvolvida uma sequência didática com base no método proposto por Lima LD e Corrallo MV (2019). A partir da utilização de trechos do livro "As Crônicas de Nárnia", assim como o primeiro filme da trilogia, aplicamos questionários a fim de realizar um paralelo entre o que se apresenta na obra e o que se espera do discurso científico. De acordo com nossos resultados, pudemos concluir que o uso da literatura como atividade didática para aquisição do conhecimento científico é bastante favorável e promissor para o ensino da física moderna, visto que essas obras possuem uma linguagem mais clara quando comparados aos textos encontrados em livros didáticos. No entanto, em tal prática interdisciplinar, que visa abandonar o método tradicional, vale ressaltar o desafio em manter o equilíbrio entre a cultura humanista e a cultura física.
\end{abstract}

Palavras-chave: Física e literatura, Ensino de física, Interdisciplinaridade.

\begin{abstract}
The present work aims to demonstrate the didactic potential in the articulation between physics and literature through an interdisciplinary methodology as stimulating pedagogical intercession in physics classes for high school students of a state public school. To this end, a didactic sequence was developed based on the method proposed by Lima LD and Corrallo MV (2019). From the use of excerpts from the book "The Chronicles of Narnia", as well as the first film of the trilogy, we apply questionnaires in order to make a parallel between what is presented in the work and what is expected of scientific discourse. According to our results, we were able to conclude that the use of literature as a didactic activity for the acquisition of scientific knowledge is very favorable and promising for the teaching of modern physics, since these works have a clearer language when compared to the texts found in textbooks. However, in this interdisciplinary practice, which aims to abandon the traditional method, it is worth emphasizing the challenge of maintaining the balance between humanist culture and physical culture.
\end{abstract}

Keywords: Physics and literature, Physical education, Interdisciplinarity.

\footnotetext{
${ }^{1}$ Secretaria de Educação e da Ciência e Tecnologia da Paraíba (SEECT-PB), Cacimba de Dentro - Paraíba.
} *E-mail: rafael.silva@professor.pb.gov.br 
Resumen: El presente trabajo tiene como objetivo demostrar el potencial didáctico en la articulación entre la física y la literatura a través de una metodología interdisciplinaria como estimular la intercesión pedagógica en las clases de física para los estudiantes de secundaria de una escuela pública estatal. Para ello, se desarrolló una secuencia didáctica basada en el método propuesto por Lima LD y Corrallo MV (2019). A partir del uso de extractos del libro "Las Crónicas de Narnia", así como de la primera película de la trilogía, aplicamos cuestionarios con el fin de hacer un paralelismo entre lo que se presenta en la obra y lo que se espera del discurso científico. Según nuestros resultados, pudimos concluir que el uso de la literatura como actividad didáctica para la adquisición de conocimientos científicos es muy favorable y prometedor para la enseñanza de la física moderna, ya que estas obras tienen un lenguaje más claro en comparación con los textos que se encuentran en los libros de texto. Sin embargo, en esta práctica interdisciplinaria, que pretende abandonar el método tradicional, vale la pena destacar el reto de mantener el equilibrio entre la cultura humanista y la cultura física.

Palabras clave: Física y Literatura, Enseñanza de la física, Interdisciplinariedad.

\section{INTRODUÇÃO}

$\mathrm{Na}$ busca por solucionar a crise no ensino de física, diversos esforços no que diz respeito a metodologias de ensino vêm sendo feitos. Nessa perspectiva, elementos da cultura popular, tais como filmes de ficção científica, leitura de literaturas de divulgação científica, literatura fantástica e até mesmo de cientistas com estilos mais literários, têm sido considerados como abordagem interdisciplinar para serem trabalhados em sala de aula (LIMA LD e RICARDO EC, 2015).

Essa perspectiva se alicerça na pergunta principal que direciona o ensino de física no ensino médio: Para que ensinar física? Nessa etapa da vida escolar, a física deverá cumprir um papel cultural, no sentido de construção humana ao longo da história, e o aspecto de compreensão do mundo (BRASIL, 2002).

Portanto, longe de se ensinar a física para quem será físico, nessa etapa ela deve destinar-se aos alunos que não serão físicos e que, possivelmente, essa será a única oportunidade de acesso formal a esse conhecimento. Isso não significa, no entanto, deixar o formalismo e rigor matemático e experimental, mas também não significa descartar o caráter histórico, filosófico e cultural desta ciência básica (LIMA LD e RICARDO EC, 2015; LIMA LD e RICARDO EC, 2017).

Com base nisso, Piassi LP (2009), constatou que a utilização de contos de ficção científica não é apenas um simples motivador de interesse. Tal metodologia desperta nos alunos não só o interesse por debate de ideias e aprofundamento em conceitos da física, mas também oferece conexões com o seu contexto sociocultural quando permite posicioná-lo frente à obra como um leitor crítico, reflexivo, favorecendo o desenvolvimento de habilidades cognitivas. Logo, é importante, mais do que saber onde a física funciona ou não, compreender como a física se relaciona com a cultura e como através de uma análise de obras culturais se pode tirar conclusões e novas perspectivas para problemas reais da vida dos alunos (PIASSI LP e PIETROCOLA M, 2007; PIASSI LP, 2013).

Nesse contexto, este trabalho busca a utilização da literatura fantástica, assim como outros elementos da cultura popular como filmes, séries de ficção científica e histórias em quadrinhos no ensino de física. Nossa escolha se dá pela alta aceitação do gênero entre os jovens. Autores como J.J.R. Tolkin e C.S. Lewis estão entres os mais lidos do mundo e suas popularidades cresceram com filmes relacionados às suas obras. Somando-se a isso, o tema do uso de literatura na física tem despertado o interesse dos pesquisadores e mostrado resultados que comprovam sua efetividade no processo ensino-aprendizagem (LIMA LD e RICARDO EC. 2015; LIMA LD e CORRALLO MV, 2019; LIMA LD, et al, 2017; LIMA LD e RICARDO EC, 2015; LIMA LD e RICARDO EC, 2019).

Apresentamos, portanto, neste trabalho, uma proposta de ensino que faça a união entre a física e a arte, mais especificamente, um relato da aplicação da literatura fantástica no ensino de física. Desse modo, mostraremos que entre a arte e os temas científicos, há uma relação com os diversos aspectos da cultura humana que possibilitam o estabelecimento de relações e conjecturas. 


\section{RELATO DE EXPERIÊNCIA}

Os resultados apresentados nesse artigo fazem parte do recorte de um conjunto de aulas para uma disciplina eletiva aplicada durante um semestre letivo, mais precisamente entre fevereiro e julho de 2018 . 0 projeto foi aplicado em uma escola estadual em um município do estado da Paraíba. Nesse modelo de escola, a cada semestre são ofertadas disciplinas eletivas onde os alunos sugerem temas de seus interesses e os professores fazem uma transposição interdisciplinar. Um dos temas mais requisitados para compor a disciplina eletiva seria uma disciplina que envolvesse séries de TV, leituras, histórias em quadrinhos e outros elementos de cultura popular.

Neste trabalho, utilizamos a metodologia de indicadores da interface Física-Literatura como produto da convergência existente nas pesquisas que tratam do tema. Os indicadores são: (1) motivação de base afetiva-volutiva; (2) presença de conceitos físicos na literatura utilizada; (3) tratamento e conversão dos conceitos presentes na leitura para os conceitos físicos objetivos de ensino (LIMA LD e CORRALLO MV, 2019).

Aproveitando do tema proposto pelos alunos, em colaboração com a professora de Português, propomos uma disciplina que envolvesse a Física e seu papel de influência na cultura mundial. Deste modo, foi dado ênfase à iniciativa e protagonismo dos alunos na construção de seu conhecimento (BRASIL, 2002; BRASIL, 2017). A defasagem quanto ao domínio de matemática básica e interpretação de texto também foram constatadas por meio um teste diagnóstico da turma. A turma da disciplina eletiva foi composta por alunos das três séries do ensino médio, totalizando 32 alunos inscritos na disciplina e, de início, muito empolgados com a proposta apresentada.

Na primeira etapa, os alunos foram convidados a ler trechos do livro "As Crônicas de Nárnia" (LEWIS CS, 2009). Como não seria viável ter um exemplar para cada um, a melhor alternativa para abordar a literatura foi utilizando esse método. O primeiro filme da trilogia "As Crônicas de Nárnia" foi exibido em sala de aula. Nessas etapas preliminares, os alunos foram incentivados a debater todas as referências quanto às noções de tempo e espaço no filme. A escolha dessa obra está relacionada ao fato de corresponder às exigências do método proposto por Lima LD e Corrallo MV (2019), especificamente o item (1), no que diz respeito à relação motivadora de base afetiva-volutiva, visto que é uma obra muito popular para o público-alvo.

Os alunos citam o fato de os tempos entre a Terra e Nárnia correrem de formas diferentes e o fato de os garotos não envelhecerem quando voltam à Terra. Com esse primeiro debate, foi possível constatar que os alunos já tinham pseudoconceitos de relatividade devido ao contato com outros filmes de ficção cientifica, inclusive abordando as possibilidades de viagem no tempo, como "De Volta Para o Futuro", "Exterminador do Futuro" e o seriado "The Flash". Deste modo, o item (2) do método de indicadores também é satisfatório, pois a obra literária contém conceitos físicos motivadores para os alunos.

Nas 5 aulas seguintes, após concluir a exibição do filme, os alunos foram submetidos ao conteúdo de relatividade referente ao ensino médio, com aula expositiva e dialogada e resolução de exercícios. $O$ conceito físico principal utilizado para gerar a discussão e coleta de respostas dos alunos encontra-se na dilatação temporal que surge naturalmente como consequência dos dois postulados da teoria especial da relatividade de Einstein, que são:

(1) "Todas as leis da natureza são as mesmas em todos os sistemas de referência que se movam com velocidade uniforme."

(2) "A velocidade de propagação da luz no espaço livre tem o mesmo valor para todos os observadores, não importando o movimento da fonte ou do observador, ou seja, a rapidez de propagação da luz é uma constante." (HEWITT PG, 2002, p. 598)

Como consequência desses dois postulados temos, naturalmente, a dilatação temporal. Conceito estranho à física newtoniana. Para sistemas de referências diferentes no espaço podemos escrever: 


$$
t=\frac{t_{0}}{\sqrt{1-\frac{v^{2}}{c^{2}}}}
$$

Onde na to é o tempo próprio medido no sistema de referência que se move com o relógio e o tempo t é medido no outro sistema de referência que se move relativo ao primeiro sistema, c é a velocidade da luz no vácuo e v em relação ao observador. Assim, os relógios funcionam mais lentamente no sistema em movimento. Isso não acontece por defeito nos relógios (aparelhos de medida do tempo), mas sim devido à natureza do tempo. A teoria da relatividade é amplamente comprovada com vários experimentos e aplicações tecnológicas.

Após essas aulas, os alunos leram um trecho do livro de Lewis CS (2009, p. 112) "Crônicas de Nárnia: o leão, a feiticeira e o guarda-roupa", que deu base ao roteiro do filme. Foi entregue uma cópia para cada aluno. Nesse trecho, Lúcia, uma das protagonistas, entra em um guarda-roupa mágico dotado da capacidade de transportá-la para o mundo de Nárnia. No entanto, quando retorna à Terra, a protagonista nota que apesar de ter passado um logo período no mundo mágico, na Terra transcorreram poucos segundos.

Foi explicado aos alunos que os livros da série foram escritos entre 1949 e 1964. Logo, nessa época, a teoria da relatividade de Einstein já gozava de popularidade. Em vista disso, não seria absurdo dizer que Lewis foi, de certa forma, influenciado pela teoria, assim como afirma Zanetic J (2005) sobre a influência da física na cultura e vice-versa.

Durante os debates realizados com os discentes foi possível verificar por meio de uma observação e relato oral que, após a aplicação da sequência didática, eles foram capazes de distinguir, em sua maioria, as referências relacionadas à teoria de Einstein sobre a relatividade e sua influência na produção artística. Os alunos se mostraram habilitados para expor as diferenças entre ficção e realidade. Por fim, muitos se dizem motivados em estudar física, e outros relataram que passaram a prestar mais atenção às aulas teóricas e experimentais. Os discentes ainda apontaram a diminuição da repulsa que sentiam pela disciplina onde antes era pouco atrativa, por ser mais voltada para cálculos e desconectada com temáticas de seus interesses.

\section{DISCUSSÃO}

As percepções dos alunos sobre o texto e em consonância com suas primeiras sínteses após as aulas teóricas de física indicam aferir que houve a transformação do conhecimento prévio do aluno sobre o tema da relatividade. Portanto, em parte, ocorreu uma reconfiguração dos conceitos primitivos de viagem no tempo e seus clichês recorrentes na cultura popular. A apresentação coletiva dos resultados e o nível das respostas confirmam a aquisição de novos conhecimentos, conforme aponta a teoria da aprendizagem significativa de Ausebel DP (2000) construíram seu conhecimento prévio de senso comum em conceitos mais elaborados de física.

Os alunos foram convidados a debaterem e transcreverem suas percepções sobre a física presente no trecho do livro, assim como no filme. É possível aferir que a transposição entre literatura em física foi efetiva, pois apresentaram o paradoxo dos gêmeos em contraposição com o trecho do livro. Os postulados da relatividade foram para explicar o fenômeno temporal no qual a personagem Lúcia se encontrou, ou seja, os alunos passaram a entendem que a natureza do tempo depende da velocidade da luz constante como um dos postulados de Einstein.

Outro fato constatado na interação foi que ao tentarem significar a obra para um sentido físico, eles relembraram algumas comprovações experimentais da relatividade com o fato de partículas subatômicas chegarem na superfície da Terra mesmo sendo contrário ao tempo de vida dessas partículas numa explicação newtoniana do fenômeno. É necessário ressaltar que, para cumprir o objetivo de ensino, a literatura escolhida deverá levar a consolidação de aprendizagem. Em outras palavras, o aluno deverá 
encontrar pela abstração a conexão entre a os conceitos de física presentes nos mais variados contextos da cultura humanísticas com conceitos físicos. Com isso, há uma transposição conceitual da linguagem semiótica com abstração para a compressão de um fenômeno (LIMA LD e CORRALLO, 2019).

A compressão das limitações físicas de viagens temporais na velocidade da luz que, em teoria, possibilitariam algo parecido com o que acontece em Nárnia na vida real foi alvo de discussão, sendo que a maioria dos alunos chegaram à conclusão de sua impossibilidade física por causa da grande quantidade de energia que seria necessária, o que reforça o ponto (2) da abordagem de Lima LD e Corrallo MV (2019) para a interface física-literatura, ou seja, a presença de conceitos físicos na literatura escolhida foi percebida.

A Base Nacional Comum Curricular (BNCC), tem como uma de suas diretrizes, garantir a contextualização dos conhecimentos, articulando as dimensões do trabalho, da ciência, da tecnologia e da cultura (BRASIL, 2020). Em outras palavras, o conhecimento científico deve fazer sentido ao aluno e a escola deve ser um meio de conexão entre saberes científicos, tecnológicos e culturais. Como sugerido por Zanetic J (2005), na busca de uma obra para aplicar como ferramenta no ensino de física, deve-se buscar os autores que utilizam conceitos científicos ou método científico.

A conexão física-literatura foi bem compreendida pelos alunos, possibilitando entenderem as vantagens de se conhecer conceitos físicos para compreender a realidade cultural que os cerca. Essa conclusão dos alunos, reforça e está de acordo com as diretrizes propostas nos Parâmetros Curriculares Nacionais, no que diz respeito ao papel cultural da física, e que a escola deve fazer essa transposição e assegurar o acesso da população a uma parte dos conhecimentos produzidos (BRASIL, 2002). Aqui, nos deparamos com a mesma conclusão de Lima LD, et al. (2017), pois tais interações onde se respeita a opinião do aluno e suas conclusões não seriam possíveis em uma aula tradicional onde se dá muita ênfase a cálculos e formalismos matemáticos. Neste caso, existiu a interação entre a cultura científica e a cultura humanística como apresentado por Snow CP (1995), que alerta para a importância de não se desenvolver em separado os dois conhecimentos, caso contrário, corremos o risco de desenvolver uma sociedade que não seja capaz de pensar seus problemas com sabedoria.

Em suma, a utilização de literatura e filmes como ferramenta geradora de debates e transformadora de conceitos científicos se mostrou efetiva como apresentado pelos resultados. É importante ressaltar que, existe um risco inerente que é o da analogia presente nas leituras, já que a analogia literária não deve ser consolidada como conceito físico. Por isso, deve-se ter uma metodologia para trabalhar física-literatura e isso significa abandonar o modo tradicional de ensinar, dando sentido aos conceitos científicos. Outro ponto interessante é que há também o perigo de valorizar a cultura humanista e deixar de lado a cultura física. Logo, a relação física-literatura é uma ferramenta didática que visa preencher lacunas da física tradicional e não a substituir.

\section{AGRADECIMENTOS}

O autor agradece, em especial, ao Dr. Luís Gomes de Lima por sua valorosa contribuição por meio de sugestões e indicações durante a realização desse trabalho.

\section{REFERÊNCIAS}

1. AUSUBEL DP. Aquisição e retenção de conhecimentos: Uma perspectiva cognitiva. Lisboa. Paralelo Editora LDA, 2000; $227 \mathrm{p}$.

2. BRASIL. Ministério da Educação, Secretaria de Educação Média e Tecnológica. Orientações Educacionais Complementares aos Parâmetros Curriculares Nacionais (PCN): Ciências da Natureza, Matemática e suas Tecnologias. Brasília: Ministério da educação, 2002.

3. BRASIL. Base Nacional Comum Curricular (BNCC). Ministério da Educação, Secretaria de Educação Continuada, Alfabetização, Diversidade e Inclusão; Secretaria de Educação Profissional e Tecnológica. Conselho Nacional de Educação; Câmara de Educação Básica, 2017. 
4. LEWIS CS. Crônicas de Nárnia: o leão, a feiticeira e o guarda-roupa. Trad. Silêda Steuernagel Paulo Mendes Campos. 2nd ed. São Paulo: WMF Martins Fontes, 2009; 112p.

5. LIMA LD, RICARDO EC. Física e Literatura: uma revisão bibliográfica. Caderno Brasileiro de Ensino de Física, 2015; 32(3), 577-617.

6. LIMA LD, CORRALLO MV. Trinta anos de física também é cultura: apresentação de estratégias didáticas para o ensino da interface física-literatura. Atas do XXIII SNEF (Simpósio Nacional de Ensino de Física), 2019.

7. LIMA LD, et al. Professor, por que eu tenho que estudar física? A física e literatura como promotora de sentidos em processos argumentativos. Atas do XXII SNEF (Simpósio Nacional de Ensino de Física), 2017.

8. LIMA LD, RICARDO EC. A literatura como ferramenta didática no ensino de Mecânica Quântica para o Ensino Médio. Atas do XXI SNEF (Simpósio Nacional de Ensino de Física), 2015.

9. LIMA LD, RICARDO EC. O Ensino da Mecânica Quântica no nível médio por meio da abstração científica presente na interface Física-Literatura. Caderno Brasileiro de Ensino de Física, 2019; 36(1), 8-54.

10. PIASSI LP, PIETROCOLA M. Ficção científica e ensino de ciências: para além do método de 'encontrar erros em filmes'. Educação e pesquisa, 2009; 35(3), 525-540.

11. PIASSI LP, PIETROCOLA M. Quem conta um conto aumenta um ponto também em física: Contos de ficção científica na sala de aula. Atas do XVII SNEF (Simpósio Nacional de Ensino de Física), 2007.

12. PIASS LP. A ficção científica e o estranhamento cognitivo no ensino de ciências: estudos críticos e propostas de sala de aula. Ciência \& Educação (Bauru), 2013; 19(1), 151-168.

13. ZANETIC J. Física e cultura. Ciência e Cultura, 2005; 57(3), 21-24. 